\title{
Neuroleptic malignant syndrome: a neuroimmunologic hypothesis
}

\author{
Rebecca E. Anglin MD, Patricia I. Rosebush MScN MD, Michael F. Mazurek MD
}

$\mathrm{N}$ euroleptic malignant syndrome is a fulminant and life-threatening toxidrome that occurs in an estimated $0.07 \%$ to $3.23 \%$ of patients treated with antipsychotic medication. ${ }^{1,2}$ Patients typically present with fever, rigidity, changes in mental status and autonomic instability, often after the start of antipsychotic medication or an increase in dosage. ${ }^{1}$ The major clinical and laboratory features used for diagnosis are summarized in Box 1. ${ }^{3}$ Possible risk factors that have been identified in case series and case-control studies include agitation, dehydration, high ambient temperature, affective illness, intramuscular injections and high doses of antipsychotic medications. ${ }^{1,2,4}$ Treatment involves cessation of antipsychotic medications and supportive care, including rehydration, cooling, antipyretics and benzodiazepines. There is evidence from case series that electroconvulsive therapy may be effective. ${ }^{5}$ The utility of dantrolene and bromocriptine is controversial. ${ }^{6}$

Despite numerous reviews and case reports, the underlying pathophysiology of neuroleptic malignant syndrome remains unknown. Some have proposed that the disorder is caused by a central hypodopaminergic state that results from antagonism to dopamine- $\mathrm{D}_{2}$ receptors induced by antipsychotic medications. ${ }^{7}$ Others emphasize the role of sympathoadrenal hyperactivity. ${ }^{8}$ Both hypotheses, however, are supported only by circumstantial evidence, and neither has been tested in welldesigned studies with adequate sample sizes. More importantly, both fail to account adequately for key features of neuroleptic malignant syndrome and do not explain what triggers its onset at a particular point in time.

We previously reported that neuroleptic malignant syndrome is associated with a profound and precipitous decrease in serum iron levels, which is a key feature of the immunologic cascade known as the acute phase response. ${ }^{9}$ We now propose a neuroimmunologic hypothesis for neuroleptic malignant syndrome, based on direct evidence that the acute phase response is activated in this toxic drug reaction. This hypothesis accounts for all of the essential features of the disorder, and has important therapeutic implications.

\section{The acute phase response}

The acute phase response is a complex physiologic reaction that classically develops in response to tissue injury (Figure 1). Cytokines are released by macrophages and monocytes, leading to the production of acute phase proteins by the liver, and a host of neuroendocrine, hematopoietic, metabolic and biochemical

\section{Key points}

- Neuroleptic malignant syndrome is an acute and lifethreatening reaction to antipsychotic medications.

- There is growing evidence that the immunologic cascade known as the acute phase response may play a role in development of neuroleptic malignant syndrome.

- We propose a neuroimmunologic hypothesis to explain the pathophysiology of neuroleptic malignant syndrome and suggest several mechanisms by which the acute phase response may be activated.

- This hypothesis may have substantial implications for treatment.

changes. ${ }^{10,11}$ The chief cytokines involved are interleukin-1, interleukin-6, tumour necrosis factor $\alpha$, interferon- $\gamma$ and transforming growth factor $\beta$. These cytokines in turn stimulate the hepatic production or suppression of acute phase proteins. Substances that increase during inflammation are termed positive acute phase reactants and include C-reactive protein, serum amyloid A protein, $\alpha-1$ acid glycoprotein, haptoglobin, complement components, $\alpha-1$ antichymotrypsin, ferritin and fibrinogen. Substances that decrease during inflammation are termed negative acute phase reactants and include iron, albumin and transferrin. The role of acute phase reactants in inflammation is complex and, in some cases, poorly understood. Some reactants are proinflammatory and initiate or amplify the inflammatory reaction through such processes as recognition and binding of foreign pathogens, and activation of the complement system. Other reactants are anti-inflammatory and diminish the inflammatory process by acting as antioxidants and promoting wound healing.

On a clinical level, the acute phase response involves a host of systemic responses to cytokines and acute phase proteins. ${ }^{10,11}$ Fever is induced by cytokines acting through prostaglandin $\mathrm{E}_{2}$ to raise the temperature set point in the hypothalamus. Other homeostatic changes associated with the acute phase reaction include somnolence, anorexia, elevated cortisol and increased secretion of catecholamines by the adrenal gland. Hematopoietic responses include leukocytosis, thrombocytosis and anemia. Metabolic changes range from

From the Department of Psychiatry \& Behavioural Neurosciences (Anglin, Rosebush, Mazurek); and the Division of Neurology, Department of Medicine (Mazurek), McMaster University, Hamilton, Ont.

CMAJ 2010. DOI:10.1503/cmaj.091442 
Box 1: Clinical and laboratory features of neuroleptic malignant syndrome*

- The development of severe muscle rigidity and elevated temperature associated with the use of neuroleptic medication.

- Two or more of the following: diaphoresis, dysphagia, tremor, incontinence, changes in level of consciousness ranging from confusion to coma, mutism, tachycardia, elevated or labile blood pressure, leucocytosis, laboratory evidence of muscle injury (e.g., elevated creatine phosphokinase).

- The symptoms are not due to another substance (e.g., phencyclidine) or a neurologic or other general medical condition.

- The symptoms are not better accounted for by a mental disorder (e.g., mood disorder with catatonic features).

*Adapted from the Diagnostic and statistical manual of mental disorders, 4th ed., American Psychiatric Assocation. ${ }^{3}$

decreased gluconeogenesis, increased lipogenesis and lipolysis to cachexia, muscle breakdown and osteoporosis. The classic biochemical profile of the acute phase response includes reduced serum levels of iron and zinc, increased levels of serum copper and changes in levels of enzymes such as creatine phosphokinase and lactate dehydrogenase.

Although the acute phase response is generally understood to serve a homeostatic function, ${ }^{10}$ there are several conditions in which it becomes dysregulated and fulminates, leading to severe illness, such as sepsis or heat stroke. ${ }^{12}$ We propose that a fulminant acute phase response may account for the clinical and biochemical features of neuroleptic malignant syndrome.

\section{Can the acute phase response explain the core features of neuroleptic malignant syndrome?}

The abnormal results of laboratory testing that are characteristic of neuroleptic malignant syndrome are typical of changes seen during the acute phase response. Leukocytosis and elevated muscle enzymes (particularly creatine phosphokinase) are consistently observed in both neuroleptic malignant syndrome and the acute phase response. ${ }^{10}$ We recently reported the temporal evolution of a variety of acute phase reactants in an instance of neuroleptic malignant syndrome. ${ }^{13}$ Whereas serum levels of creatine phosphokinase, erythrocyte sedimentation rate, $\mathrm{C}$-reactive protein, interleukin- $6, \alpha-1$ antichymotrypsin and fibrinogen increased, levels of serum iron and albumin declined. These results are consistent with activation of the acute phase response. Levels of positive acute phase reactants, erythrocyte sedimentation rate, C-reactive protein, interleukin-6, $\alpha-1$ antichymotrypsin, and fibrinogen peaked within 72 hours. Levels of negative acute phase reactants (i.e., albumin and serum iron), were low and gradually increased into the normal range, and this increase was coincident with clinical improvement. This temporal profile of acute phase reactants paralleled changes in temperature, creatine phosphokinase levels and the clinical course of the syndrome, sug- gesting that the acute phase response may have been directly involved in the pathophysiology of neuroleptic malignant syndrome in that particular patient.

As we reported previously, a dramatic drop in serum iron levels is consistently associated with neuroleptic malignant syndrome, and is a key feature of the acute phase response. ${ }^{9}$ Subsequent studies have replicated this finding. ${ }^{14}$ Combined with our original 26 instances, we have now measured serum iron levels in 33 patients with neuroleptic malignant syndrome. In 32 of the 33 patients, serum iron levels were low, with a mean of 5.38 (normal 13-32) $\mathrm{mmol} / \mathrm{L}$ and a standard deviation of 2.78. In each of these patients, serum iron levels returned to normal with resolution of the syndrome. In the remaining patient, the levels were at the lowest limit of normal and returned to a high-normal range with recovery.

The core clinical findings in neuroleptic malignant syndrome include fever, tachycardia, diaphoresis, unstable blood pressure, pupillary dilation and decreased mobility, all of which are classic features of the acute phase response (Table 1). Other core features (e.g., severe rigidity, staring and mutism), might result from reduced function of dopamine receptors caused by low serum iron levels associated with the acute phase response. There is strong evidence that iron is essential for the normal functioning of the dopamine $\mathrm{D}_{2}$ receptor. ${ }^{15}$ Hence, the reduction in serum iron levels that occurs in the acute phase response may result in a decreased number of functional dopamine $\mathrm{D}_{2}$ receptors in the brain. In a setting of dopamine blockade induced by antipsychotic medication, this loss of receptors may trigger an acute reduction in dopaminergic transmission mediated by $\mathrm{D}_{2}$ receptors and thereby give rise to the rigidity, staring and mutism that are characteristic of neuroleptic malignant syndrome.

\section{Proposed mechanisms for induction of the acute phase response}

We propose several mechanisms for the induction of the acute phase response in neuroleptic malignant syndrome: autoantibody production, virus-drug interaction, heat stress, muscle breakdown and psychological stress. Some of these conditions may occur in concert and may together result in the crossing of a threshold and the generation of neuroleptic malignant syndrome.

\section{Autoantibody production}

Demonstration of the acute phase response occurring in neuroleptic malignant syndrome points to involvement of the innate immune system. Additional evidence suggests there is also activation of the adaptive immune response. Patients with a history of neuroleptic malignant syndrome have been shown to have autoantibodies against neurotransmitter receptors, with indices more than four standard deviations above the mean of healthy control patients. ${ }^{16}$ The antigenic stimulus for induction of the immune response could be the antipsychotic medication itself, interacting with an endogenous protein in the brain. The example of heparin-induced thrombocytopenia may be instructive in this regard. In that disorder, patients transiently produce immunoglobulin- $\mathrm{G}$ antibodies 
against heparin bound to platelet factor IV, which activates platelets and leads to thrombocytopenia. ${ }^{17}$ As in neuroleptic malignant syndrome, heparin-induced thrombocytopenia can occur at any time during treatment with heparin, and may not recur with subsequent exposure to the medication. These characteristics provide a useful model when considering neuroleptic malignant syndrome.

\section{Virus-drug interaction}

In the last several decades, it has become apparent that viruses can interact with medications, resulting in adverse drug reactions. It is likely that the viral infection alters immune regulation, leading to decreased tolerance to the medication. We propose that neuroleptic malignant syndrome may be associated with a virus-drug interaction, in which a predisposing viral illness triggers a fulminant acute phase response to antipsychotic medication. In our original case series of neuroleptic malig- nant syndrome, $29 \%$ of patients had clinically obvious rash, ${ }^{1}$ suggesting a possible co-existing viral exanthem.

A well-known example of a virus-drug reaction is the development of maculopapular erythematous rash in virtually all patients with infectious mononucleosis treated with ampicillin. ${ }^{18}$ Most of these patients have a history of tolerating ampicillin in the past. On re-exposure to this medication in the absence of Epstein-Barr infection, they do not develop a rash, suggesting that active infection with the virus produces an interaction with the ampicillin and results in a fulminant drug reaction. Likewise, in Reye syndrome, patients develop fatty degeneration of the liver and encephalopathy when treated with acetylsalicylic acid during the course of a viral illness. ${ }^{19}$ In both of these examples, viruses and medications interact to produce an adverse drug reaction, raising the possibility that something similar may trigger neuroleptic malignant syndrome.

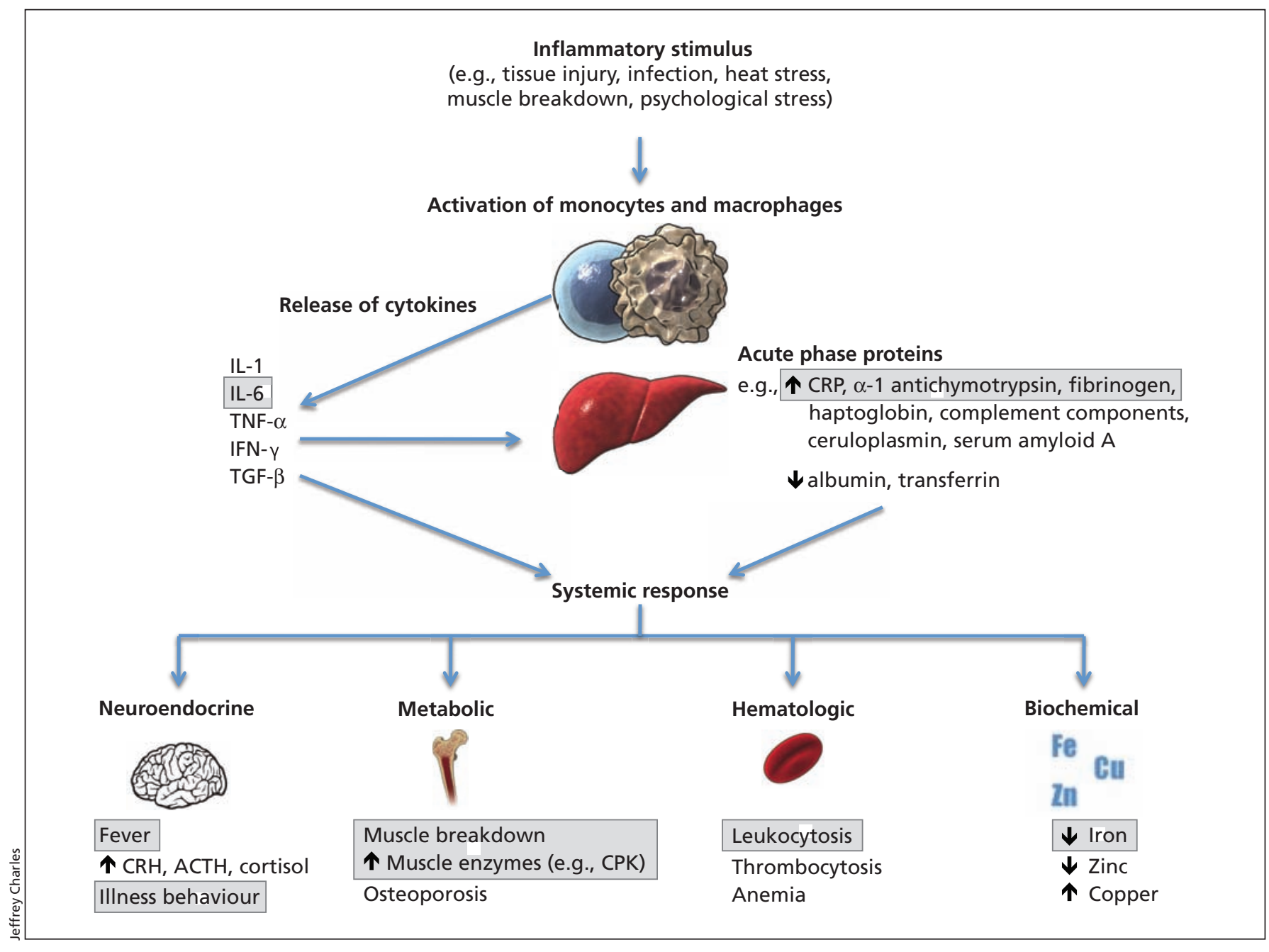

Figure 1: The acute phase response. An inflammatory stimulus results in activation of monocytes and macrophages that release cytokines. The cytokines act on the liver to stimulate production of acute phase proteins. Cytokines, together with acute phase proteins, generate a systemic response, with neuroendocrine, metabolic, hematologic and biochemical changes. Features of the acute phase response that have been directly observed and measured in neuroleptic malignant syndrome are shown in boxes. Note: $\mathrm{ACTH}=$ adrenocorticotropic hormone, $\mathrm{CPK}=$ creatine phosphokinase, $\mathrm{CRH}=$ corticotropin-releasing hormone, $\mathrm{CRP}=\mathrm{C}$-reactive protein, IFN = interferon, IL = interleukin, TGF = transforming growth factor, TNF = tumour necrosis factor. (Portions of this illustration were reproduced with permission from Lianne Friesen and Nicholas Woolridge.) 
Table 1: Comparison of the clinical features of neuroleptic malignant syndrome and the acute phase response

\begin{tabular}{|c|c|c|}
\hline System & $\begin{array}{c}\text { Neuroleptic } \\
\text { malignant syndrome }\end{array}$ & Acute phase response \\
\hline \multirow[t]{3}{*}{$\begin{array}{l}\text { Motor or } \\
\text { behavioural }\end{array}$} & $\begin{array}{l}\text { Rigidity and muscle } \\
\text { breakdown }\end{array}$ & $\begin{array}{l}\text { Myalgia and } \\
\text { muscle breakdown }\end{array}$ \\
\hline & Immobility & Decreased motor activity \\
\hline & Mutism & Withdrawal, somnolence \\
\hline \multirow[t]{5}{*}{ Autonomic } & Fever & Fever \\
\hline & Tachycardia & Tachycardia \\
\hline & Diaphoresis & Diaphoresis \\
\hline & Dilation of pupils & Dilation of pupils \\
\hline & $\begin{array}{l}\text { Elevated or unstable } \\
\text { blood pressure }\end{array}$ & $\begin{array}{l}\text { Elevated or unstable } \\
\text { blood pressure }\end{array}$ \\
\hline
\end{tabular}

\section{Heat stress}

There is considerable evidence that heat stress is capable of inducing the acute phase response. ${ }^{12}$ Most instances of neuroleptic malignant syndrome occur during the summer months in settings that lack adequate air conditioning, ${ }^{1}$ suggesting impaired regulation of body temperature. This impairment could occur through a hypothalamic failure to sense elevated temperature and activate the appropriate mechanisms for heat loss, or through direct stimulation of hypothalamic thermoregulatory centres by prostaglandins. In addition to such central mechanisms, peripheral heat may be produced (as a result of extrapyramidal side effects such as akathisia) at the same time as sweating is impaired by the anticholinergic properties of many psychotropic medications.

Several conditions associated with psychiatric illness may lower a patient's safety margin for developing neuroleptic malignant syndrome as a result of heat stress. Psychiatric patients, particularly those with schizophrenia, have abnormal thermoregulation, including elevated baseline temperature, abnormal circadian variation in body temperature and impaired tolerance to heat. ${ }^{20}$ Excess clothing, poor hydration and comorbid illness may also contribute to development of hyperthermia.

\section{Muscle breakdown}

Acute muscle injury resulting from strenuous exercise ${ }^{21}$ or myocardial infarction ${ }^{22}$ has been shown to initiate the acute phase response. Based on similarities with malignant hyperthermia, it has been proposed that patients with neuroleptic malignant syndrome may have abnormal calcium channels in skeletal muscle. ${ }^{23}$ In a series of eight patients with neuroleptic malignant syndrome, six were found to have evidence of elevated levels of free calcium in myoplasm. ${ }^{24}$ Three patients with neuroleptic malignant syndrome had a mean resting calcium level more than four times that of normal in skeletal muscle, even after recovery. ${ }^{25}$ These findings suggest that patients with neuroleptic malignant syndrome may have abnormal calcium metabolism, perhaps reflecting a distinct channelopathy. In these susceptible patients, the acute phase response may be activated in the periphery by muscle breakdown associated with dystonia, parkinsonian rigidity, akathisia or psychomotor agitation. Subsequent damage to muscle (reflected in the consistent finding of elevated levels of muscle enzymes) during neuroleptic malignant syndrome may then prolong an acute phase response.

\section{Psychological stress}

Prior to developing neuroleptic malignant syndrome, patients are often agitated, psychotic and under marked psychological stress, which is a known trigger for the acute phase response. ${ }^{26}$ An additional contributor can be catatonia, which commonly occurs immediately before neuroleptic malignant syndrome $^{27}$ and is associated with intense fear. ${ }^{28}$ Animal studies have shown that acute stress, such as immobilization and inescapable shock, increases indices of interleukin-1b in the brain, ${ }^{29}$ suggesting that the stress-induced acute phase response can be activated centrally.

\section{Therapeutic implications}

Currently, the treatment of neuroleptic malignant syndrome is largely supportive and involves cessation of antipsychotic medications, rehydration, cooling, judicious administration of antipyretics, low-dose heparin when immobility is prolonged, and benzodiazepines to control agitation and anxiety.

The possibility that the acute phase response plays a role in the pathophysiology of neuroleptic malignant syndrome has important therapeutic implications. Given that prostaglandin $E_{2}$ is a critical mediator in the function of many cytokines, prostaglandin $\mathrm{E}_{2}$ inhibitors (e.g., salicylates, nonsteroidal anti-inflammatory drugs) may be effective therapeutic agents in addition to providing symptomatic relief. These agents inhibit cyclo-oxygenase, the rate-limiting enzyme in the production of prostaglandins from arachidonic acid. Identification of the acute phase response as the biologic substrate of neuroleptic malignant syndrome could potentially lead to the development of novel treatments, including agents that block leukocyte activation or modify cytokine action. If the acute phase response in neuroleptic malignant syndrome is found to be triggered either by autoantibodies or a virus-drug interaction, this finding could also lead to preventive and therapeutic strategies, such as closer monitoring of patients treated with neuroleptics during viral illness and use of immune-modulating agents.

\section{Limitations}

Although we have presented a rationale for how the acute phase response can result in all of the core features of neuroleptic malignant syndrome and described the current evidence that supports our theory, more studies are required to test our hypothesis. We have shown a consistent, precipitous decline in serum iron levels in a large number of patients with neuroleptic malignant syndrome, pointing to activation of the acute phase response. However, the full profile of acute phase reactants has been measured only in a single case. In addition, the acute phase response itself does not directly explain the 
extreme rigidity, staring and mutism that are observed in neuroleptic malignant syndrome. We have proposed that these symptoms are best explained by altered function of dopamine $\mathrm{D}_{2}$ receptors resulting from acute phase-related decreases in central iron levels and occurring in concert with dopamine receptor blockade caused by antipsychotic medication. Although we have measured only peripheral iron levels, there is strong evidence that these levels correlate with dysfunction of the central nervous system, suggesting that peripheral measures may be sufficient to support our hypothesis. ${ }^{30}$

\section{Testing our hypothesis}

Neuroleptic malignant syndrome is uncommon and requires prompt treatment, which poses problems for the study of this condition. A prospective multicentre study would be needed to allow serial measurement of acute phase reactants over the course of illness in a large number of patients. This type of study would also allow measurement of autoantibodies to both antipsychotic medications and neurotransmitters in patients with active neuroleptic malignant syndrome, as well as screening for evidence of a viral infection that might trigger a virus-drug reaction. If our neuroimmunologic hypothesis of neuroleptic malignant syndrome is supported by further study, multicentre therapeutic trials would be warranted.

This article has been peer reviewed.

\section{Competing interests: None declared.}

Contributors: Rebecca Anglin, Patricia Rosebush and Michael Mazurek contributed to the conceptualization of the article. Rebecca Anglin drafted the manuscript, and all of the authors were involved in its revision. All of the authors approved the final version of the manuscript submitted for publication.

\section{REFERENCES}

1. Rosebush P, Stewart T. A prospective analysis of 24 episodes of neuroleptic malignant syndrome. Am J Psychiatry 1989;146:717-25.

2. Strawn JR, Keck PE, Caroff SN. Neuroleptic malignant syndrome. Am J Psychiatry 2007; 164:870-6.

3. Diagnostic and statistical manual of mental disorders. 4th ed. Washington (DC) American Psychiatric Association; 2000. p. 798.

4. Keck PE, Pope HG, Cohen BM, et al. Risk factors for neuroleptic malignant syndrome: A case-control study. Arch Gen Psychiatry 1989;46:914-8.

5. Troller JN, Sachdev PS. Electroconvulsive treatment of neuroleptic malignant syndrome: a review and report of cases. Aust N Z J Psychiatry 1999;33:650-9.
6. Rosebush PI, Stewart T, Mazurek MF. The treatment of neuroleptic malignant syndrome: are dantrolene and bromocriptine useful adjuncts to supportive care? Br J Psychiatry 1991;159:709-12.

7. Henderson VW, Wooten GF. Neuroleptic malignant syndrome: A pathogenetic role for dopamine receptor blockade? Neurology 1981;31:132-7.

8. Gurrera RJ. Sympathoadrenal hyperactivity and the etiology of neuroleptic malignant syndrome. Am J Psychiatry 1999;156:169-80.

9. Rosebush PI, Mazurek MF. Serum iron and neuroleptic malignant syndrome. Lancet 1991;328:149-51.

10. Gabay C, Kushner I. Acute-phase proteins and other systemic responses to inflammation. N Engl J Med 1999;340:448-54.

11. Burger D, Dayer J. Cytokines, acute-phase proteins, and hormones. Ann N Y Acad Sci 2002;966:464-73.

12. Bouchama A, Knochel JP. Heat stroke. N Engl J Med 2002;346:1978-88.

13. Rosebush PI, Anglin RE, Richards C, et al. Neurolptic malignant syndrome and the acute phase response. J Clin Psychopharmacol 2008;28:459-61.

14. Lee S. Serum iron in catatonia and neuroleptic malignant syndrome. Biol Psychiatry 1998;44:499-507.

15. Ben-Shachar D, Finberg JPM, Youdim MBH. Effect of iron chelators on dopamine D2 receptors. J Neurochem 1985;45:999-1005.

16. Tanaka S, Matsunaga H, Kimura M, et al. Autoantibodies against four kinds of neurotransmitter receptors in psychiatric disorders. J Neuroimmunol 2003;141: 155-64.

17. Kelton JG. The pathophysiology of heparin-induced thrombocytopenia: biological basis for treatment. Chest 2005;(Suppl 2)127:9S-20S.

18. Pullen H, Wright N, Murdoch JM. Hypersensitivity reactions to antibacterial drugs in infectious mononucleosis. Lancet 1967;2:1176-8.

19. Levy M. The combined effect of viruses and drugs in drug-induced diseases. Med Hypotheses 1984;14:293-6.

20. Chong TW, Castle DJ. Layer upon layer: thermoregulation in schizophrenia. Schizophr Res 2004;69:149-57.

21. Taylor C, Rogers G, Goodman C, et al. Hematologic, iron-related and acute-phase protein responses to sustained strenuous exercise. J Appl Physiol 1987;62:464-9.

22. Griffiths JD, Camphell IJ, Woodruff IW, et al. Acute changes in iron metabolism following myocardial infarction. Am J Clin Pathol 1985;84:649-54.

23. Gurrera RJ. Is neuroleptic malignant syndrome a neurogenic form of malignant hyperthermia. Clin Neuropharmacol 2002;25:183-93.

24. Araki M, Takagi A, Higuchi I, et al. Neuroleptic malignant syndrome: caffeine contracture of single muscle fibers and muscle pathology. Neurology 1988;38:297-301.

25. Lopez JR, Sanchez V, Lopez MJ. Sarcoplasmic ionic calcium concentration in neuroleptic malignant syndrome. Cell Calcium 1989;10:223-33.

26. Deak T, Meriwether JL, Fleshner M, et al. Evidence that brief stress may induce the acute phase response in rats. Am J Physiol 1997;273(6 pt 2):R1998-2004.

27. White DA. Catatonia and the neuroleptic malignant syndrome - a single entity? Br J Psychiatry 1992;161:558-60.

28. Rosebush PI, Hildebrand A, Furlong BG, et al. Catatonic syndrome on a general psychiatric ward: frequency, clinical presentation and response to lorazepam. $J$ Clin Psychiatry 1990;51:357-62.

29. Nguyen KT, Deak T, Owens SM, et al. Exposure to acute stress induces brain interleukin-1b protein in the rat brain. $J$ Neurosci 1998;18:2239-47.

30. Allen RP, Earley CJ. The role of iron in restless legs syndrome. Mov Disord 2007; 22:S440-8.

Correspondence to: Dr. Patricia I. Rosebush, St. Joseph's

Healthcare, Fontbonne Building Room F416, 50 Charlton Ave. E,

HamiltonON L8N 4A6; rosebush@hhsc.ca 